\title{
A facile strategy to construct high-performance nanofiltration membrane by synergy of graphene oxide and polyvinyl alcohol
}

Lufang Shen a,b, Quanling Xie ${ }^{\text {b,d,*, }}$, Zhuan Hong b,d, Chenpu Wu ${ }^{\text {a,b }}$, Tong Yu ${ }^{\text {b,d }}$, Hua Fang ${ }^{\text {b,d }}$, Ying Xiong ${ }^{\mathrm{c}}$, Guoliang Zhang e, Yinghua Lu ${ }^{\mathrm{a}, *}$, Wenyao Shao ${ }^{\mathrm{a}, *}$

${ }^{a}$ Department of Chemical and Biochemical Engineering, College of Chemistry and Chemical Engineering, Xiamen University, Xiamen 361005, PR China

${ }^{\mathrm{b}}$ Technology Innovation Center for Exploitation of Marine Biological Resources, Third Institute of Oceanography, Ministry of Natural Resources, Xiamen 361005, PR China

${ }^{c}$ Guangdong Provincial Key Laboratory of Soil and Groundwater Pollution Control, School of Environmental Science and Engineering, Southern University of Science and Technology, Shenzhen 518055, PR China

${ }^{\mathrm{d}}$ Fujian Collaborative Innovation Center for Exploitation and Utilization of Marine Biological Resources, Xiamen, 361005, PR China

e Institute of Oceanic and Environmental Chemical Engineering, State Key Lab Breeding Base of Green Chemical Synthesis Technology, Zhejiang University of Technology, Hangzhou, 310014, PR China

\footnotetext{
* Corresponding author.
}

E-mail address: qlxie@tio.org.cn (Q. Xie), ylu@xmu.edu.cn (Y. Lu), wyshao@xmu.edu.cn (W. Shao) 

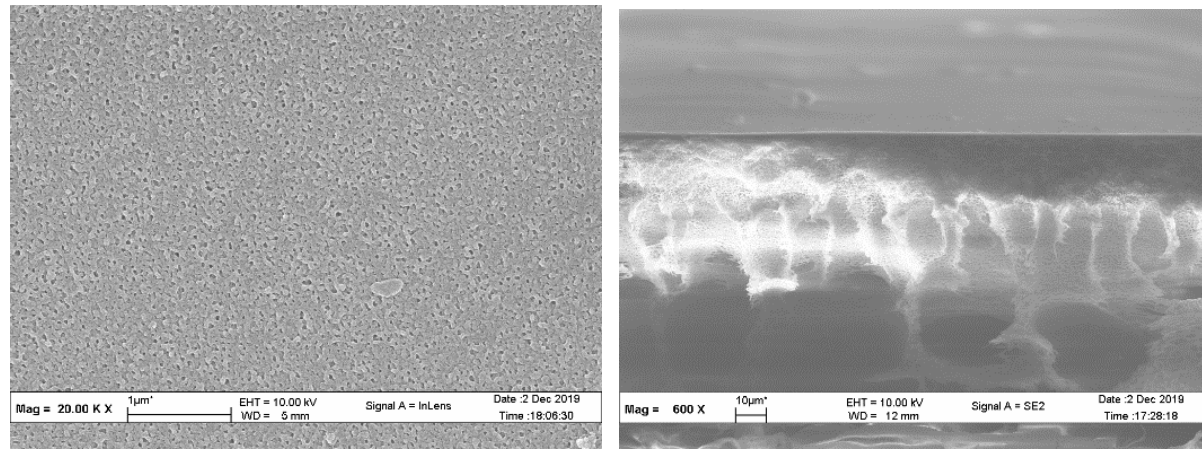

Fig.S1. Surface and cross-sectional SEM images of PSU support.

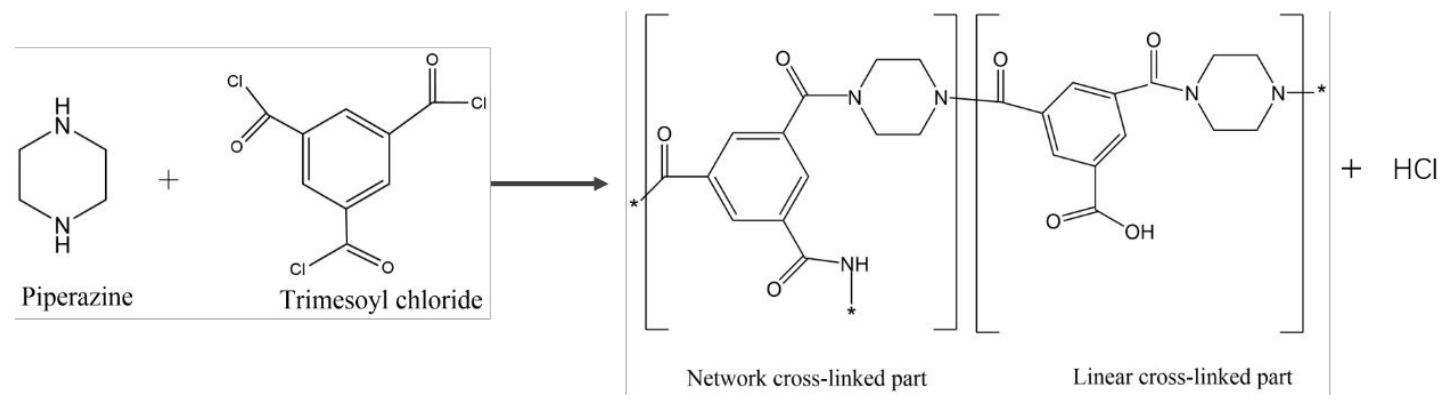

Fig. S2. Interfacial polymerization of PIP and TMC and polymers formed with linear cross-linked part and network cross-linked part.

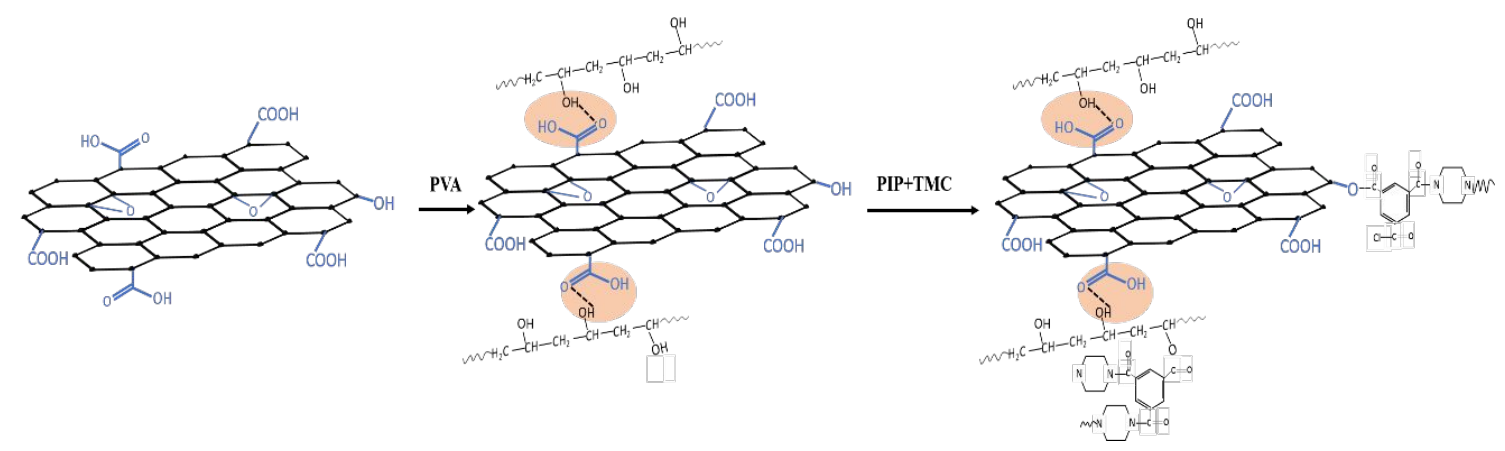

Fig. S3. The schematic illustration of reaction mechanism between PIP, GO, PVA, and TMC. 


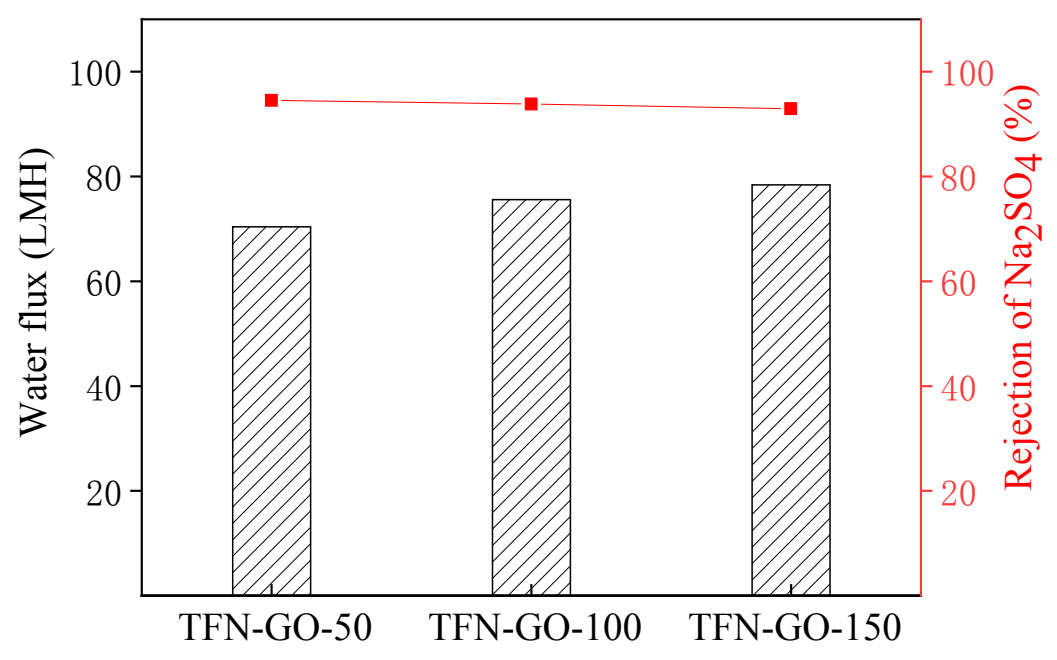

Fig. S4. Water fluxes and $\mathrm{Na}_{2} \mathrm{SO}_{4}$ rejections of the TFN-GO membranes $\left(\mathrm{Na}_{2} \mathrm{SO}_{4}\right.$ concentration: $2 \mathrm{~g} / \mathrm{L}$, TMP: $0.55 \mathrm{MPa}$ ) 
Table S1 Parameters of related ions ${ }^{[1]}$.

\begin{tabular}{lcccc}
\hline \multicolumn{1}{c}{ Ions } & $z$ & $D\left(10^{-3} \mathrm{~mm}^{2 \cdot} \mathrm{s}^{-1}\right)$ & $r_{\mathrm{s}}(\mathrm{nm})$ & Hydrated radius $(\mathrm{nm})$ \\
\hline $\mathrm{Na}^{+}$ & 1 & 1.333 & 0.183 & 0.36 \\
$\mathrm{Mg}^{+}$ & 2 & 0.706 & 0.345 & 0.43 \\
$\mathrm{Cl}^{-}$ & -1 & 2.032 & 0.120 & 0.33 \\
$\mathrm{SO}^{2-}$ & -2 & 1.065 & 0.229 & 0.38 \\
\hline
\end{tabular}

Table.S2 Comparison of the performance of NF membranes prepared in this study with recent reports of NF membranes with graphene oxide and other nanoparticles

\begin{tabular}{lclcl}
\hline Membrane & $\begin{array}{c}\text { Water flux } \\
(\mathrm{LMH} / \mathrm{bar})\end{array}$ & Feed solution & $\begin{array}{c}\text { Salt rejection } \\
(\%)\end{array}$ & Ref. \\
\hline $\mathrm{NF} / \mathrm{rGO} / \mathrm{TiO} 2$ & 6.1 & $\mathrm{Na}_{2} \mathrm{SO}_{4}\left(1 \mathrm{~g} \mathrm{~L}^{-1}\right)$ & 94 & {$[2]$} \\
$\mathrm{NF} / \mathrm{GO}$ & 6.9 & $\mathrm{Na}_{2} \mathrm{SO}_{4}\left(1 \mathrm{~g} \mathrm{~L}^{-1}\right)$ & 92.62 & {$[3]$} \\
$\mathrm{NF} / \mathrm{GO}-\mathrm{COCl}$ & 3.77 & $\mathrm{Na}_{2} \mathrm{SO}_{4}\left(1 \mathrm{~g} \mathrm{~L}^{-1}\right)$ & 97.1 & {$[4]$} \\
ZIF-8 & 14.9 & $\mathrm{Na}_{2} \mathrm{SO}_{4}\left(1 \mathrm{~g} \mathrm{~L}^{-1}\right)$ & 93.1 & {$[5]$} \\
$\mathrm{NF90}$ & 9.69 & $\mathrm{Na}_{2} \mathrm{SO}_{4}\left(1 \mathrm{~g} \mathrm{~L}^{-1}\right)$ & 98.6 & {$[6]$} \\
PIP-GO TFN & 18.1 & $\mathrm{MgSO}_{4}\left(2 \mathrm{~g} \mathrm{~L}^{-1}\right)$ & 91.2 & {$[7]$} \\
PEI/PIP TFC NF & 18.2 & $\mathrm{Na}_{2} \mathrm{SO}_{4}\left(1 \mathrm{~g} \mathrm{~L}^{-1}\right)$ & 77.4 & {$[8]$} \\
GO/EDA-5cycles & 4.7 & $\mathrm{MgCl}_{2}\left(1 \mathrm{~g} \mathrm{~L}^{-1}\right)$ & 97.6 & {$[9]$} \\
BPPO/EDA/GO & 4.1 & $\mathrm{Na}_{2} \mathrm{SO}_{4}\left(1 \mathrm{~g} \mathrm{~L}^{-1}\right)$ & 56.2 & {$[10]$} \\
TFN-PG-100 & 15.3 & $\mathrm{Na}_{2} \mathrm{SO}_{4}\left(2 \mathrm{~g} \mathrm{~L}^{-1}\right)$ & 97.6 & This work \\
& & $\mathrm{MgSO}_{4}\left(2 \mathrm{~g} \mathrm{~L}^{-1}\right)$ & 91.7 & This work \\
\hline
\end{tabular}




\section{Reference}

[1] Q.L. Xie, S.S. Zhang, Z. Hong, H.J. Ma, C.R. Liu, W.Y. Shao, Effects of Casting Solvents on the Morphologies, Properties, and Performance of Polysulfone Supports and the Resultant Graphene Oxide-Embedded Thin-Film Nanocomposite Nanofiltration Membranes, Ind. Eng. Chem. Res., 57 (2018) 16464-16475.

[2] N. Zhang, B. Jiang, L. Zhang, Z. Huang, Y. Sun, Y. Zong, H. Zhang, Low-pressure electroneutral loose nanofiltration membranes with polyphenol-inspired coatings for effective dye/divalent salt separation, Chemical Engineering Journal, 359 (2019) 1442-1452.

[3] R. Hu, Y. He, C. Zhang, R. Zhang, J. Li, H. Zhu, Graphene oxide-embedded polyamide nanofiltration membranes for selective ion separation, Journal of Materials Chemistry A, 5 (2017) 25632-25640.

[4] P. Wen, Y. Chen, X. Hu, B. Cheng, D. Liu, Y. Zhang, S. Nair, Polyamide thin film composite nanofiltration membrane modified with acyl chlorided graphene oxide, Journal of Membrane Science, 535 (2017) 208-220.

[5] J.Y. Zhu, L.J. Qin, A. Uliana, J.W. Hou, J. Wang, Y.T. Zhang, X. Li, S.S. Yuan, J. Li, M.M. Tian, J.Y. Lin, B. Van der Bruggen, Elevated Performance of Thin Film Nanocomposite Membranes Enabled by Modified Hydrophilic MOFs for Nanofiltration, ACS Appl. Mater. Interfaces, 9 (2017) 1975-1986.

[6] W. Zhao, H. Liu, N. Meng, M. Jian, H. Wang, X. Zhang, Graphene oxide incorporated thin film nanocomposite membrane at low concentration monomers, Journal of Membrane Science, 565 (2018) 380-389.

[7] L. Miao, Y. Yang, Y. Tu, S. Lin, J. Hu, Z. Du, M. Zhang, Y. Li, Chiral resolution by polysulfone-based membranes prepared via mussel-inspired chemistry, Reactive and 
Functional Polymers, 115 (2017) 87-94.

[8] W.X. Fang, L. Shi, R. Wang, Mixed polyamide-based composite nanofiltration hollow fiber membranes with improved low-pressure water softening capability, Journal of Membrane Science, 468 (2014) 52-61.

[9] Y. Zhang, S. Zhang, J. Gao, T.S. Chung, Layer-by-layer construction of graphene oxide (GO) framework composite membranes for highly efficient heavy metal removal, Journal of Membrane Science, 515 (2016) 230-237.

[10] N. Meng, W. Zhao, E. Shamsaei, G. Wang, X.K. Zeng, X.C. Lin, T.W. Xu, H.T. Wang, X.W. Zhang, A low-pressure GO nanofiltration membrane crosslinked via ethylenediamine, Journal of Membrane Science, 548 (2018) 363-371. 